\title{
VIBRATION DIAGNOSTICS OF POWER EQUIPMENT BEFORE COMMISSIONING
}

\author{
Mikolay Hruntovich ${ }^{1, *}$, Deniz Moroz $^{2}$, Alexey Panfilov ${ }^{1}$, Yegor Zhuk ${ }^{1}$, and Ekaterina Mikhailova ${ }^{3}$ \\ ${ }^{1}$ Sukhoi State Technical University of Gomel, Prospect Octiabria, 48, 246746, Gomel, Republic of Belarus \\ ${ }^{2}$ State Enterprise "Research Institute Belgiprotopgaz", Domashevskij lane,11a, Minsk, Republic of Belarus \\ ${ }^{3}$ Kazan State Power Engineering University, str. Krasnoselskaya, 51, 420066, Kazan, Russia
}

\begin{abstract}
The article describes how to use vibration diagnostics to detect defects in the installation of new equipment and to determine the quality of repairs based on vibroacoustic characteristics. Especially relevant is the vibration diagnostics of rolling bearings before installation on the mechanism. If there are errors in the calculations of the magnetic system of synchronous generators, vibration occurs, which is not eliminated during operation. Design errors, poor assembly of pumping units, asynchronous motors, power transformers cause increased vibration, which leads to damage to the specified equipment. The main reasons causing the vibration of the transformer tank are considered and the frequency range from 50 to $6000 \mathrm{~Hz}$ is determined on which these defects can occur. Full-scale experiments of the vibration of the transformer tank have shown that certain defects can occur in a narrow frequency band. The practical application vibration diagnostics of the pump unit describes in this article. Technical diagnosis of pumping units of the sewer station was carried out for various included composition. The results obtained made it possible to exclude reverse hydraulic impacts as a possible cause of the failure of the pump unit.
\end{abstract}

\section{Introduction}

Quality control of purchased equipment is controlled by various regulatory documents $[1,2]$. The necessary conditions for performing maintenance and repair of equipment is the presence of a modern system of maintenance and repair at the enterprise, which includes: products - objects of maintenance and repair; diagnostic tools [2]; performers (organizations, specialists); documentation (design, including operational and repair, regulatory, organizational, technological, etc.) establishing requirements for components of the maintenance and repair system and the relationship between them [3].

Efficiency of product maintenance and repair can be improved due to: improvement of product design as object of maintenance and repair; Improvement of the product maintenance and repair strategy in accordance with operational data on its reliability; testing of operational and maintenance documents; providing diagnostic control of both the unit as a whole and its individual units during repairs; development of normative documents based on modern scientific and technological developments; qualification of maintenance and repair contractors; application of modern methods of technical diagnostics of various equipment.

\section{Research results}

Consider vibration diagnostics of electric machines and rolling bearings.

\subsection{Vibration diagnostics of rolling bearings before installation on the mechanism}

Current studies have shown that about $60 \%$ of bearings delivered to enterprises are low-quality due to increased vibration. A vibration speed of $2.8 \mathrm{~mm} / \mathrm{s}$ was adopted to reject new bearings. Measurement of vibration acoustic characteristics of bearings on vibration test bench showed that $40 \%$ of tested group of bearings in frequency range from 5 to $600 \mathrm{~Hz}$ and $60 \%$ of bearings in the range of $600 \mathrm{~Hz}-5000 \mathrm{~Hz}$ do not pass by vibration level. Typical defects of new rolling bearings are: different dimensions of rolling bodies; not roundness of bodies of swing; ovality of an internal ring; Defects of inner and outer rings. Vibration frequencies of bearings in the range of up to $600 \mathrm{~Hz}$ for the listed defects can be calculated by formulas [3, 4].

\subsection{Technical diagnostics of magnetic vibration of synchronous generators}

During technical diagnostics of magnetic vibration, acceleration vibration sensor is placed on synchronous

* Corresponding author: gruntovich@tut.by 
generator housing. The magnetic vibration of the housing of a synchronous generator with a capacity of 60 MW of thermal power plant at the frequency of $100 \mathrm{~Hz}$ was more than $4.5 \mathrm{~mm} / \mathrm{s}$. At this vibration level, longterm operation is not allowed. After the conducted regime studies, it was suggested that errors were made in the calculations of the magnetic system. This type of synchronous generator was subsequently discontinued.

\subsection{Technical diagnostics of magnetic vibration of asynchronous motors}

Under operating conditions, the magnetic vibration of asynchronous motors is practically not diagnosed.

\subsubsection{Technical diagnostics of uneven gap between rotor and stator of asynchronous electric motors}

The vibration value of the motor housing depends on the induction value in the air gap:

$$
\begin{gathered}
B_{\varepsilon}(\alpha, t)=F_{\mathrm{cp}}(\alpha, t) \lambda_{\varepsilon}(\alpha, t), \\
F_{\mathrm{cp}} \cos \left(\omega_{1} t-p \alpha\right) \times \\
\times\left(\lambda_{0}+\lambda_{1 \varepsilon} \cos \left(\omega_{1}(1-s) \frac{p_{\varepsilon}}{p}-p_{\varepsilon} \alpha\right)\right),
\end{gathered}
$$

or:

$$
\begin{gathered}
B_{\varepsilon}(\alpha, t)=F_{\mathrm{cp}} \lambda_{0} \cos \left(\omega_{1} t-p \alpha\right)+ \\
+\cos \left(\left(\frac{p_{\varepsilon}}{p}(1-s) \pm 1\right) \omega_{1} t-\left(p_{\varepsilon} \pm p\right) \alpha\right),
\end{gathered}
$$

where $F_{\mathrm{cp}}$ - the resultant magnetic moving force of stator and rotor in the air gap; $\omega_{1}-$ the angular frequency; $p$ - number of pole pairs; $\alpha$ - angular coordinate in geometric radians; $\lambda_{\varepsilon}-$ the magnetic conductivity in the gap; $\lambda_{0}-$ constant component of magnetic conductivity; $\lambda_{1 \varepsilon}-$ amplitude of the first harmonic of conductivity; $s$ - engine slip; $p_{\varepsilon}-$ magnetic conductivity poles.

The above expression shows that induction waves are formed in the air gap, having different speeds, polarities, and amplitudes. The speed of the induction wave determines the frequency of the magnetic gravity forces, and the order or polarity - moment arm causing deformation of stator ring. Forces with a large moment arm, i.e. polarity, are most dangerous. Table 1 shows numerical values of frequencies and order of magnetic forces arising due to eccentricity in asynchronous engine with different numbers of pole pairs $\mathrm{p}$ for medium sliding modes $\mathrm{s}=0.05$ and $\mathrm{f}_{1}=50 \mathrm{~Hz}$.

\begin{tabular}{|c|c|c|c|c|c|c|c|c|c|c|c|c|}
\hline \multirow{3}{*}{ Group } & \multicolumn{6}{|c|}{$p_{\varepsilon}=1$ (shaft deflection) } & \multicolumn{6}{|c|}{$p_{\varepsilon}=2$ (rotor ellipsis) } \\
\hline & \multicolumn{2}{|c|}{$p=1$} & \multicolumn{2}{|c|}{$p=2$} & \multicolumn{2}{|c|}{$p=3$} & \multicolumn{2}{|c|}{$p=1$} & \multicolumn{2}{|c|}{$p=2$} & \multicolumn{2}{|c|}{$P=3$} \\
\hline & $f_{r}$ & $r$ & $f_{r}$ & $r$ & $f_{r}$ & $r$ & $f_{r}$ & $r$ & $f_{r}$ & $r$ & $f_{r}$ & $r$ \\
\hline I & 100 & 2 & 100 & 4 & 100 & 6 & 100 & 2 & 100 & 4 & 100 & 6 \\
\hline \multirow{4}{*}{ II } & 148 & 3 & 124 & 5 & 116 & 7 & 195 & 4 & 148 & 6 & 132 & 8 \\
\hline & 48 & 1 & 24 & 1 & 16 & 1 & 95 & 2 & 48 & 2 & 32 & 2 \\
\hline & 48 & 1 & 24 & 1 & 16 & 1 & 95 & 2 & 48 & 2 & 32 & 2 \\
\hline & 52 & 1 & 76.5 & 3 & 84.5 & 5 & 95 & 0 & 52.5 & 2 & 68.5 & 4 \\
\hline III & 95 & 2 & 48 & 2 & 32 & 2 & 192 & 4 & 95 & 4 & 63.5 & 4 \\
\hline \multirow{2}{*}{ IV } & 195 & 4 & 148 & 6 & 132 & 8 & 280 & 6 & 195 & 8 & 163 & 10 \\
\hline & $4 \div 6$ & 0 & 52 & 2 & 68.5 & 4 & 90 & 2 & $4 \div 6$ & 0 & 36.5 & 2 \\
\hline
\end{tabular}

Table 1. Values of frequencies and order of magnetic forces arising due to eccentricity in asynchronous engine with different numbers of pole pairs $\mathrm{p}$ for medium sliding modes $\mathrm{s}=$ 0.05 and $\mathrm{f} 1=50 \mathrm{~Hz}$.

In case of rotating eccentricity, more dangerous forces of the order $r=1$ are generated at frequencies equal to or close to unbalanced ("reverse"). Magnetic vibrations at these frequencies are superimposed on the unbalance vibrations, increasing the vibration level at this frequency.

Only eccentricity explains presence of significant magnetic vibrations at frequencies close to frequency of stator winding splicing network $50 \mathrm{~Hz}$.

When the numbers of poles of the main magnetic field and conductivity $p=p_{\varepsilon}$ are matched, magnetic forces are generated at low frequencies $f_{r}=4 \div 6 \mathrm{~Hz}$. These forces have zero order $r=0$ and can cause pulsation in the air gap. As a result, amplitude modulation of high-frequency magnetic (e.g. tooth) vibrations occurs. The oscillations at this frequency are close to the own oscillations of the machine mounted on the shock absorbers.

\subsubsection{Technical diagnostics of stator winding splicing}

During long-term operation of asynchronous electric motors, stator winding insulation dries and wedges in slots perform their function poorly. During operation of electric motor winding vibrates. Vibration frequency is calculated by formulas:

$$
f_{s t 1}=Z_{p} f_{p} ; f_{s t 2}=2 Z_{p} f_{p},
$$

where $Z_{p}-$ number of slots in the stator; $f_{p}-$ speed of rotation of the rotor.

Vibration sensor is installed along the stator barrel circumference. The larger the measurement points, the more accurate the sector of the stator barrel with the weakened split winding is determined.

\subsection{Vibrodiagnosing of power transformers}

Vibration of the transformer tank housing can be caused by the following reasons: magnetostrictive vibration appearing up to $700 \mathrm{~Hz}$; attenuation of winding and magnetic core pressing (loss of dynamic resistance), which manifests in the whole frequency range; damage 
to the branch lines in $110 \mathrm{kV}$ transformers and formation of bubbles in oil, which appears in the frequency range from 700 to $1500 \mathrm{~Hz}$; resonance of frequencies of individual transformer elements measured at frequency above $1500 \mathrm{~Hz}[1,2]$.

From the established practice, losses of dynamic resistance of windings and magnetic circuit of transformers are determined by the total vibration level in the frequency range of 50-1000 Hz [3, 4, 5]. Vibration diagnostics of two new autotransformers of the railway traction substation showed that there are defects that cause vibration in the range of 1000-5000 Hz. Vibration spectra are shown in Figure 1: in autotransformer № 1 the limit vibration level at frequency $2800 \mathrm{~Hz}$, and in autotransformer № 2 the limit vibration at frequencies 3500-3600 Hz. These vibrations are due to a defect in the installation of the autotransformers. It should be noted that in both transformers in the zone of increased vibration there are registered partial discharges, which in turn cause release of hydrogen $\left(\mathrm{H}_{2}\right)$ in transformer oil.

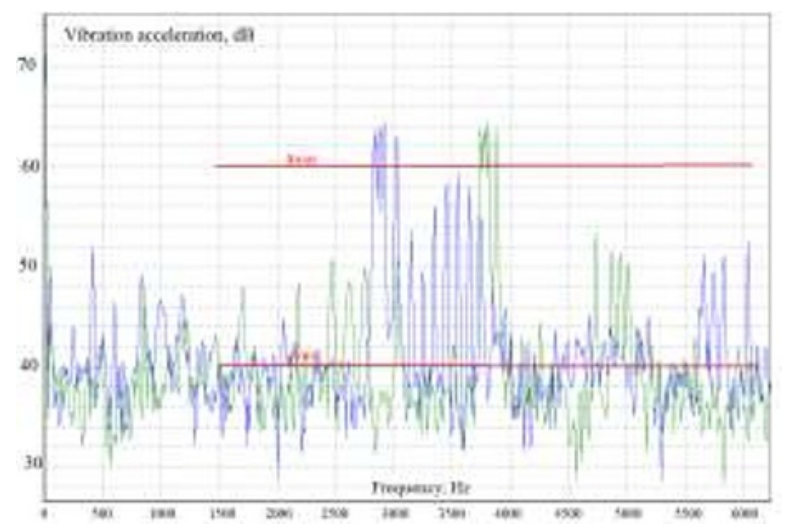

Fig. 1. Comparison of vibration levels of two autotransformers in the tank bottom of two autotransformers: green autotransformer №. 1, blue - autotransformer № 2 .

\subsection{Vibrodiagnosing of pump units}

Let's consider an example of practical application of the results of vibration diagnostics to identify the causes of failure of a pump unit with a capacity $P_{n o m}=95 \mathrm{~kW}$ of pump units at a sewage station [6]. The pump unit that failed suffered damage to the impeller due to the selfunscrewing of the impeller mounting bolt. After replacement of shaft and impeller of pump unit the situation was repeated and repeated damage to impeller and shaft occurred (Figure 2). The supplier concluded that the pump failure was caused by a hydraulic shock due to an incorrectly selected pump unit and operating conditions that did not correspond to the pump. It was decided to measure vibroacoustic characteristics of Hidrostal snail pump with capacity of $75 \mathrm{~kW}$ installed instead of failed pump unit for three possible modes of pump station operation: 1st mode - parallel operation of two pump units; 2nd mode - parallel operation of three pump units; 3rd mode - serial disconnection of parallel operating pump units. In this case, removal of vibration acoustic characteristics of three possible modes of operation made it possible to confirm or refute the occurrence of reverse hydraulic impacts. Vibration acoustic characteristics were removed using a multichannel computer vibration acoustic diagnostic measuring complex.

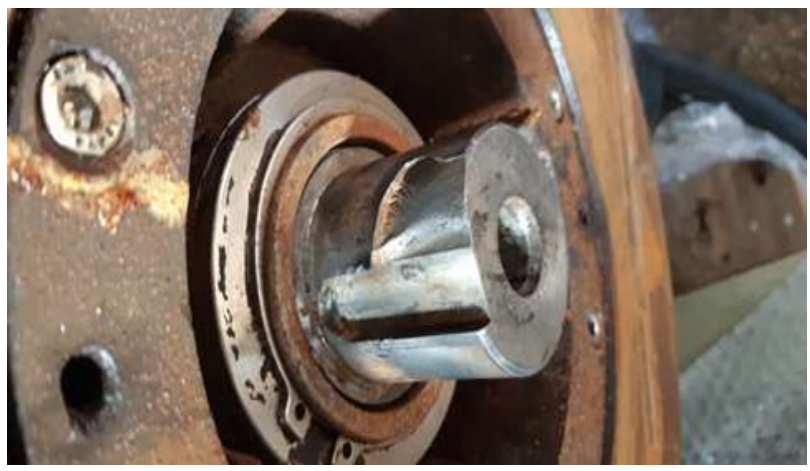

Fig. 2. Electric motor shaft damages by pump impeller.

According to GOST 32106-2013 "Monitoring and diagnostics of machines. Monitoring of the state of hazardous production equipment. Vibration of centrifugal pump and compressor units" [7, 8, 9] limit value of vibration speed of new pump equipment is 4.1 $\mathrm{mm} / \mathrm{s}$, which corresponds to vibration acceleration of $78.5 \mathrm{~dB}$. As a result of the operation, it was found that for the first mode the maximum vibration acceleration was $72 \mathrm{~dB}$, or vibration speed $-1.9 \mathrm{~mm} / \mathrm{s}$ (Figure 3 ).

\section{Conclusions}

For the second mode, the maximum vibration acceleration was $74 \mathrm{~dB}$, or vibration speed was 2.4 $\mathrm{mm} / \mathrm{s}$. For the third mode, the vibration of the pump did not change, which indicates that there is no effect of the change in the composition of the station equipment on the vibration of each of them $[10,11]$.

Thus, thanks to the technical diagnosis of the pump units, the cause of failure of the pump unit due to hydraulic shocks in the system was eliminated.

The use of vibrodiagnostic control for power equipment and its individual units and elements can significantly increase the efficiency of the existing system of maintenance and repair:

- studies have shown that vibration diagnostics of rolling bearings before installation on the mechanism allows you to identify factory defects and avoid accidents with significant material damage. The magnetic vibration of induction motors causes not only accelerated wear of the insulation, but also damage the bearings;

- the measurements of vibration of the transformer tanks made it possible not only to determine the defects causing them, but also the frequencies at which these defects can occur. In this case, partial discharges with the release of combustible gases may occur. The conducted vibration diagnostics of the tank of two autotransformers (without turning them off) of the railway traction substation made it possible to establish installation defects;

- vibrodiagnostics of pumping units of the sewer station made it possible to evaluate all possible operating 
modes of the pumping station in order to exclude the reason for the failure of the pumping unit due to water hammer in the system.

Deep knowledge of the physical bases of vibration of different objects allows to solve practical problems of vibration diagnostics of these objects.

\section{References}

[1] GOST 16504-81 The state system of testing products, Product test and quality inspection. General terms and definitions (in Russian)

[2] GOST 24297-2013 Verification of purchased products, Organization and methods of control (in Russian)

[3] Nic.Vas. Hruntovich, Improving the maintenance and repair system, Energy strategy, 21-26 (in Russian) (2013)

[4] Nic.Vas. Hruntovich, I.V. Petrov, D.V. Kirdishev, Hypocycloid vibration frequency of rolling bearings, Modern issues of energy saving and energy efficiency in technical systems, Tambov, 25-27 April 2016, 288-289 (2016)

[5] N.V. Hruntovich, et al., Vibration diagnostic of electric motor roller bearings, E3S Web of Conferences 124, EDP Sciences (2019)

[6] Nic.Vas. Hruntovich, Installation, commissioning and use of electrical equipment (Minsk, 271, 2013) (in Russian)

[7] Y.I. Gracheva, V.N. Petrov, N.V. Russova, D.V. Samuilov, G.P. Svintsov, I.V. Protosovitsky, On modeling on a single-winding forced valve electromagnet in a circuit with a ballast resistor. E3S Web of Conferences 124, 02012, SES-2019, 5-9 (2019)

[8] Nic.Vas. Hruntovich, O.V. Chaus, On some methods of obtaining information in conditions of uncertainty: deterministic and stochastic aspects, GSTU Bulletin 3, 99-107 (2012)

[9] Nic.Vas. Hruntovich, O.V. Fedorov, D.R. Moroz, B.B. Tretyakov, P.M. Kolesnikov, Analysis of problematic issues in the operation of oil-filled transformers, Energy and Management 3, 96, 2-6 (2017)

[10] N.V. Hruntovich, A.A. Kapanski, D. Baczynski, G.V. Vagapov, O.V. Fedorov, Optimization of a variable frequency drive pump working on a water tower, E3S Web of Conferences 124, 05060, EDP Sciences (2019)

[11] N.V. Hruntovich, E.A. Zhuk, Improving the reliability of technical diagnostics of power oilfilled transformers is the basis of their durability and reliability, GSTU Bulletin 4, 60-68 (2019)

[12] B.T. Holm-hansen, R.X. Gao, Structural design and analysis for a sensor-integrated ball bearing / Finite elements in analysis and design, 257-270 (2000)

[13] A.V. Barkov, A.V. Barkova, A.Y. Azovtsev, Monitoring and diagnosis of rotary machines by vibration $(159,2000)$

[14] Y.P. Aslamov, I.G. Davidof, Sparse wavelet vibrating signal decomposition for pattern recognition task, PSU Bulletin 12, 12-23 (2018)

[15] A.V. Doronichev, K.V. Konstantinov, Expert system for vibrodiagnosting rotating bearings status and repair planning in connection with current technical conditions on the basis of wavelet-technologies introduction in the field railway transportation objects, Modern technologies, System analysis, Modeling 1, 29, 92-95 (2011) 